\title{
Identification of Drag Parameters of Flow in High Permeability Materials by U-Tube Method
}

\author{
R. Drelich • M. Pakuła • M. Kaczmarek
}

Received: 14 March 2013 / Accepted: 18 September 2013 / Published online: 4 October 2013

(C) The Author(s) 2013. This article is published with open access at Springerlink.com

\begin{abstract}
The identification procedure of linear and nonlinear drag parameters of flow of liquid in high permeability materials by U-tube method is presented. The experimental technique is based on control of pressure in liquid oscillating in the U-tube including porous material and direct computer data acquisition. The macroscopic model which takes into account inertial forces, gravity, and interaction of oscillating liquid with porous material and U-tube walls is elaborated. The drag parameters are determined numerically for porous foams by fitting model predictions to experimental data. The methodology incorporates calibration of the U-tube system without sample of porous material, which is a necessary step to determine independently parameters of interaction of liquid with tube walls.
\end{abstract}

Keywords Permeability · Nonlinear drag coefficient · Identification · U-tube · Oscillations

\section{Introduction}

Quantitative description of fluid flow through porous materials requires knowledge of drag parameters present in models of flow and responsible for mechanical interaction between fluid and porous solid. In the case of linear model (for low or moderate flow velocity), the drag is uniquely represented by permeability of porous material and viscosity of fluid, while in the nonlinear model (for high flow velocity), an additional drag parameter (non-Darcy, Forchheimer or inertia coefficient) must be known (Hassanizadeh and Gray 1987; Zeng and Grigg 2006). An estimation of the two drag parameters is most frequently performed by steady flow methods using (i) extrapolation of experimental results to zero velocity limit (for determining permeability) and infinite velocity limit (for determining inertia coefficient) or (ii) finding the parameters at once by best fit of model prediction to experimental data throughout, e.g., the least-square method (Antohe et al. 1997). When porous materials have

R. Drelich · M. Pakuła · M. Kaczmarek $(\varangle)$

Institute of Mechanics and Applied Computer Science, Kazimierz Wielki University,

Kopernika 1, 85-074 Bydgoszcz, Poland

e-mail: mkk@man.poznan.pl 
high permeability, as for example foams or trabecular bones, the identification of the drag parameters can be related to relatively significant uncertainty in permeability measurements (Antohe et al. 1997; Bhattacharya et al. 2002; Sharma and Siginer 2010) which results from limited precision of controlling pressure gradient and/or flow velocities as well as from boundary effects, i.e., mainly friction loss in conduits. The measurements become yet more problematic if only small samples of homogeneous materials are available (e.g., trabecular bone samples).

In this paper, a method of identification of two drag parameters of flow in porous materials, based on oscillating flow in the U-tube system, is considered. Because of inertia (proportional to mass of liquid in U-tube) and gravity (the restorative force), due to transfer between the potential and kinetic energy of liquid, the liquid disturbed in U-tube can perform oscillatory movement. The motion is dumped due to viscous interaction of liquid with porous material and U-tube walls. The form of motion (oscillatory or not) from disturbed to equilibrium states depends on the relative contribution of dissipation as compared to the total mechanical energy of liquid. The dissipation has two components: in tubes and in porous sample. For high permeability materials, the drag forces between liquid and porous material are moderate enough to observe oscillatory motion of liquid before it reaches the equilibrium. Previously the U-tube experimental system for studies porous materials has been considered by Rehbinder (1992). However, the linear model of flow was taken into account, and the measurements of liquid level in U-tube were made with a resistance gage. The method proposed in the present paper extends the model and uses as the alternative experimental solution the measurement of oscillations of liquid pressure, which is more available source of data than measurement of oscillations of liquid level.

\section{Experimental System}

In Fig. 1, the experimental system used to measure local pressure changes during liquid oscillations in U-tube with a sample of porous material is shown. The system consists of a glass tube of constant internal radius $r=0.02 \mathrm{~m}$, radius of curvature $R=0.083 \mathrm{~m}$ and length of the branches of about $1 \mathrm{~m}$. At one branch, where the curved tube connects with the straight one, between cross-sections 3 and 4 in Fig. 1, a sample of tested porous material of length $\mathrm{L}$ is placed.

The sample is circumferentially jacketed in elastic silicon tube which prevents sidewall flow. At the opposite branch of the system (cross-section 2 in Fig. 1), there is an analog manometer $(\mathrm{P})$ which continuously measures the pressure during the test. Then the signal acquired from the manometer is digitized by a analog-to-digital converter (A/D) with sampling rate of $1 \mathrm{kHz}$ and a resolution of 12 bits (oscilloscope HS4-10, TiePie Engineering) and transferred via USB to PC for further offline analysis. The third- order low-pass digital Butterworth filter implemented in the MATLAB environment was used to remove noise from experimentally measured signals. As the main frequency of the measured signals was about $0.77 \mathrm{~Hz}$ and the peak of the Gaussian noise was about $16 \mathrm{~Hz}$, the cutoff frequency applied to the measurement signal was $5 \mathrm{~Hz}$.

The differences in the amplitudes between the raw and filtered signals in the useful part of data are smaller than $0.5 \%(\sim 0.28 / 0.01)$. The signal-to-noise ratio amounts $15.5 \mathrm{~dB}$.

The denoised signals are used in the identification procedure. The initial levels of free surface of liquid columns are forced by pumping air above liquid (by the air pump) in one of the branches while the end of the second branch remains open. Then, the registration of liquid pressure starts, and the air pressure is released by opening the solenoid 


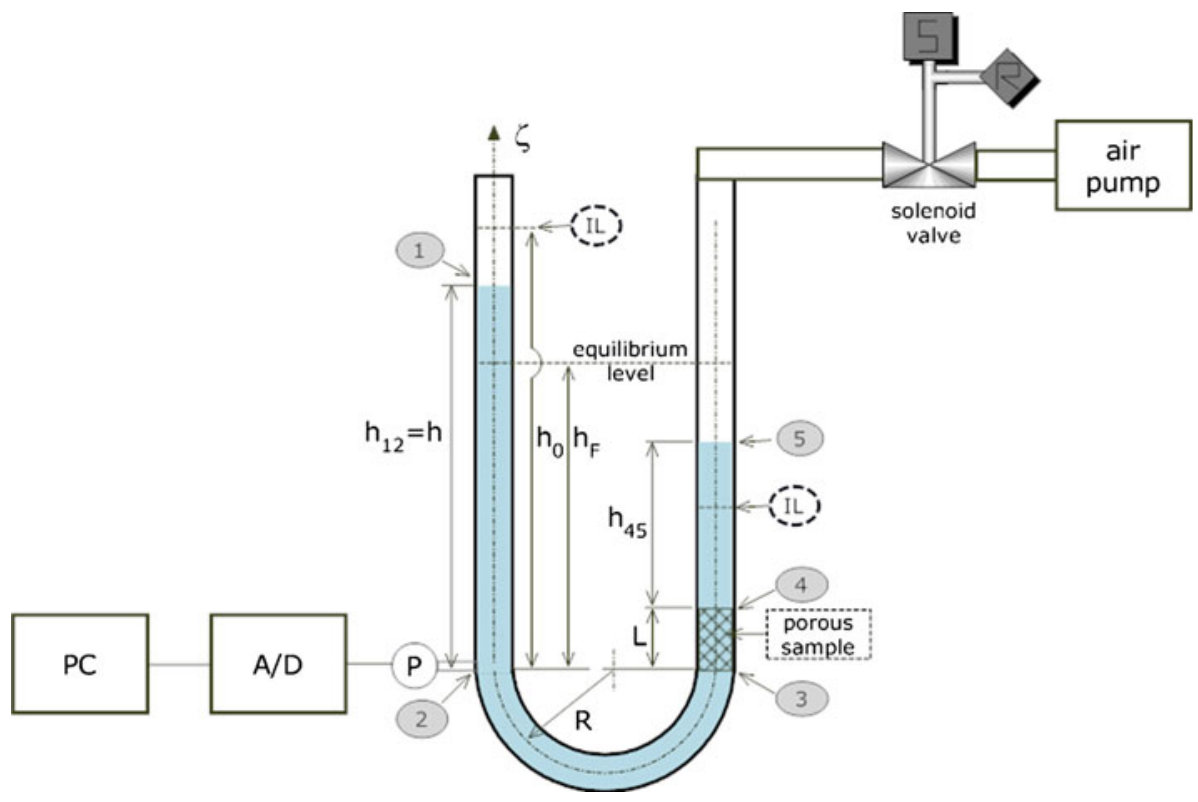

Fig. 1 Schematic of experimental system used to control pressure changes associated with motion of liquid in U-tube containing a porous sample; $P$ manometer, $A / D$ analog-to- digital converter, $P C$ personal computer, $I L$ initial liquid level

valve. The acquisition of pressure covers oscillations and terminates when the liquid reaches equilibrium.

\section{Mathematical Model}

The mathematical macroscopic model describing motion of liquid in U-tube system including a sample of porous material, shown in Fig. 1, is obtained by considering the balance of linear momentum along axis $\zeta$ of the three liquid columns bounded by cross-sections 1-2, 2-3, and $4-5$ and liquid which fills the porous material between cross-sections $3-4$. The equations incorporating inertial forces, forces due to pressure, gravity forces and drag or friction forces both between water and tube walls as well as between liquid and porous sample for these four compartments write:

$$
\begin{aligned}
& \rho h_{12} S \frac{\mathrm{d} v_{12}}{\mathrm{~d} t}=\left(p_{2}-p_{1}\right) S-\rho g h_{12} S+\tau_{12} \\
& \rho \pi R S \frac{\mathrm{d} v_{23}}{\mathrm{~d} t}=\left(p_{3}-p_{2}\right) S+\tau_{23} \\
& n \rho L S \frac{\mathrm{d} v_{34}}{\mathrm{~d} t}=n\left(p_{4}-p_{3}\right) S+n \rho g L S+\tau_{34} \\
& \rho h_{45} S \frac{\mathrm{d} v_{45}}{\mathrm{~d} t}=\left(p_{5}-p_{4}\right) S+\rho g h_{45} S+\tau_{45}
\end{aligned}
$$

where $\rho$ is mass density, $S$ is surface area of the internal cross-section of the tube, $v_{12}, v_{23}$, and $v_{45}$ are velocities of the considered liquid columns, $v_{34}$ denotes the average pore liquid velocity, $n$ stands for porosity, $p_{1}, p_{2}, p_{3}, p_{4}$, and $p_{5}$ are pressures at the specified points 
of the flow path, $g$ denotes the gravity acceleration, $\tau_{12}, \tau_{23}$, and $\tau_{45}$ are interaction forces between liquid and tube walls, and $\tau_{34}$ denotes the interaction between liquid and porous sample. Assuming incompressibility of liquid, the velocities of liquid columns are identical and the pore velocity is related to the other velocities by porosity, i.e., selecting the coordinate $h_{12}=h$ as the independent variable, we have

$$
v_{12}=v_{23}=v_{45}=\frac{\mathrm{d}}{\mathrm{d} t} h, \quad v_{34}=\frac{1}{n} \frac{\mathrm{d}}{\mathrm{d} t} h
$$

Taking into account the balance of mass of water, we may write that

$$
h_{12}+h_{45}+L=2 h_{F}
$$

where $h_{F}$ is the height of the column 1-2 at equilibrium.

When the balance equations (1) are summed up taking into account relations (2) and the balance of mass (3), we obtain the following equation

$$
\rho D S \frac{\mathrm{d}^{2} h}{\mathrm{~d} t^{2}}=2 \rho g\left(h_{F}-h\right) S+\tau+v
$$

where

$$
D=\pi R+2 h_{F}+L\left(\frac{1}{n}-1\right), \tau=\tau_{12}+\tau_{23}+\tau_{45} \quad \text { and } \quad v=\frac{1}{n} \tau_{34}
$$

The interaction force between liquid and tube walls $(\tau)$ was thoroughly discussed in the paper by Kaczmarek et al. (2013) where macroscopic modeling of liquid oscillations in Utube system without porous material were considered. In general, the force was assumed as composed of a dynamic interaction term (proportional to the liquid acceleration and related to the inertia of the boundary layer, the variation of the tube cross-section and the curvature of the U-tube), linear in liquid velocity term (representing viscous interactions) and nonlinear velocity term (responsible for micro-inertial interactions). Since the last term is mainly associated with turbulent flow during large oscillations in U-tubes and the oscillations for the U-tube system including porous material are significantly limited, the nonlinear interaction in the latter case will be neglected. As a result, it will be assumed that the interaction has the following form

$$
\tau=\left[-\rho\left(K_{1}-1\right) \frac{\mathrm{d}^{2} h}{\mathrm{~d} t^{2}}-K_{2} \frac{\mathrm{d} h}{\mathrm{~d} t}\right] D_{t} S
$$

where $K_{1}$ and $K_{2}$ are parameters representing the interaction of liquid for particular Utube system which must be identified in a calibration procedure (see Sect. 4), and $D_{t}=$ $\pi R+2 h_{12 F}-L$.

The interaction force between pore liquid and porous sample (neglecting friction at the sample's wall) according to the well-known nonlinear form (called also Hazen-DupuitDarcy equation) which neglects dynamic interaction (Hassanizadeh and Gray 1987; Zeng and Grigg 2006; Kaczmarek 2009) is

$$
v=-\left[n \frac{\mu}{k} q+\beta n^{2} \rho|q| q\right] L S
$$

where $q=\frac{1}{n} \frac{\mathrm{d} h}{\mathrm{~d} t}$ is the discharge velocity, $\mu$ is the liquid viscosity, $k$ and $\beta$ denotes linear and nonlinear drag parameters of flow through porous materials. The parameter $k$ is usually called permeability. The equation (6) takes into account not only classical interactions due to viscous friction but also micro-inertial or turbulent flow in porous material, which may appear 
particularly during relatively high flow velocities in highly permeable samples. Combining the balance of linear momentum (4) with the interaction forces (5) and (6), we obtain the equation for liquid level

$$
\rho D K_{1} \frac{\mathrm{d}^{2} h}{\mathrm{~d} t^{2}}=2 \rho g\left(h_{F}-h\right)-D_{t} K_{2} \frac{\mathrm{d} h}{\mathrm{~d} t}-\frac{\mu L}{k} \frac{\mathrm{d} h}{\mathrm{~d} t}-\beta \rho L\left|\frac{\mathrm{d} h}{\mathrm{~d} t}\right| \frac{\mathrm{d} h}{\mathrm{~d} t}
$$

If the solution for $h$ is found from the Eq. (7) with appropriate initial conditions, the first equation of the system (1) can be used to determine the associated with the oscillations pressure $p_{2}$ in liquid at the level of manometer with respect to atmospheric pressure

$$
p_{2}-p_{1}=h\left[\rho g+K_{2} \frac{\mathrm{d} h}{\mathrm{~d} t}+\rho K_{1} \frac{\mathrm{d}^{2} h}{\mathrm{~d} t^{2}}\right]
$$

where the interaction $\tau_{12}$ was assumed by analogy with the Eq. (5). The pressure according to Eq. (8) has contributions from gravity, viscous and inertial forces. Simulations have shown that the last two components must be incorporated to predict the proper value of pressure $p_{2}$. In the rest of this paper, the pressure difference $p_{2}-p_{1}$ will be analyzed in units of height of equivalent water column ( $\mathrm{mm} \mathrm{H}_{2} \mathrm{O}$ or shortly $\mathrm{mm}$ ).

In the developed identification procedure, the Eq. (7) is solved numerically, assuming the initial level $h_{0}$ and initial velocity equal to zero, by Runge-Kutta method within Matlab environment.

\section{Results}

\subsection{Experiments}

Two types of high porosity open cell rigid polyurethane foams (Sawbones Europe AB, Malmö) commonly used as the phantoms of trabecular bones have been studied. The materials denoted as foam $\mathrm{A}$ and $\mathrm{B}$ have densities 0.09 and $0.12 \mathrm{~g} / \mathrm{cm}^{3}$ and compressive modulus 6.2 and 18.6 $\mathrm{MPa}$, respectively. The microstructure of the materials is similar, while the porosities amount $88 \%$ (A) and $91 \%$ (B). The Fig. 2. presents the photomicrographs of the studied materials, obtained by Optical microscope Axiotech 100 (Carl Zeiss).

In order to remove the air bubbles from the pores of the material, prior to the permeability measurement, the samples were saturated with distilled water and degassed using vacuum pump.

\section{Foam A}

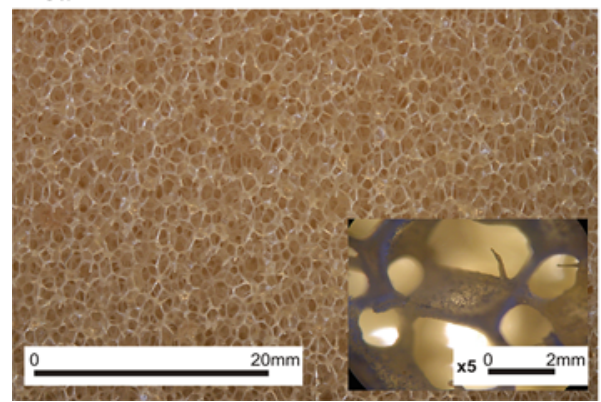

Foam B

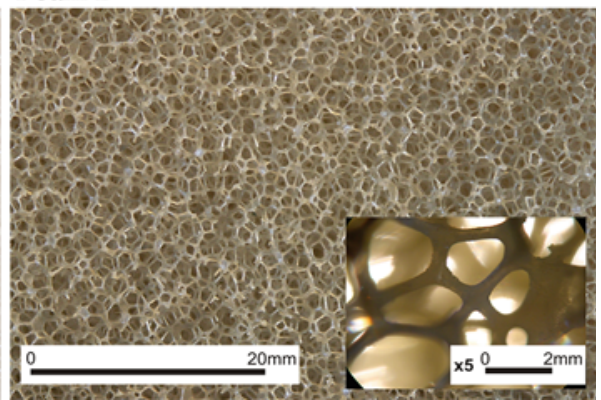

Fig. 2 Photos and photomicrographs (magnification $\times 5$ ) of tested foams A and B 


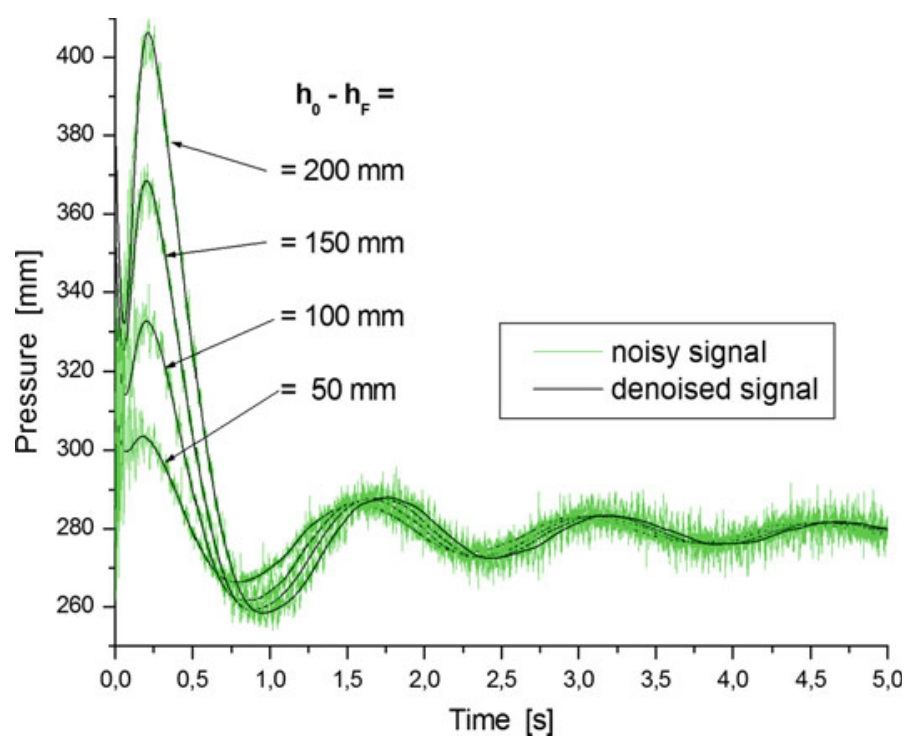

Fig. 3 Experimental results obtained for foam A showing pressure oscillations in tests with different initial height $h_{0}-h_{F}$

Then, the tests were performed in distilled water for various initial liquid levels $h_{0}$ equal to 50, 100, 150 and $200 \mathrm{~mm}$ with respect to the equilibrium level $h_{F}$. The plots in Fig. 3 show both, typical noisy (raw) signals registered from the manometer as well as the denoised signals obtained by digital filtering.

It is worth noticing that although the pressure in the first period of oscillations reaches significantly different maximum values, depending on the initial liquid level, the amplitudes in the next periods become almost identical.

\subsection{Calibration of the U-Tube System}

In order to determine the macroscopic parameters responsible for the interaction of liquid with tube walls modeling studies, experiments and identification procedure were elaborated for the U-tube system without porous material (Kaczmarek et al. 2013). The procedure was similar to the one described in the present paper and was necessary because available from the literature models of the interaction do not give satisfactory agreement with experimental data.

In general, the interaction force was assumed as composed of three components: (i) dynamic interaction term (proportional to liquid acceleration and related to inertia of boundary layer, variation of tube cross-section and curvature of the U-tube), (ii) linear with respect to the liquid velocity term (representing viscous interactions) and (ii) nonlinear with respect to the liquid velocity term (responsible for viscous and micro-inertial interactions). As a result, the model which describes the oscillations of liquid in empty U-tube reads

$$
\rho K_{1} D_{e} \frac{\mathrm{d}^{2} h}{\mathrm{~d} t^{2}}=2 \rho g\left(h_{F}-h\right)-K_{2} D_{e} \frac{\mathrm{d} h}{\mathrm{~d} t}-K_{3} D_{e}\left|\frac{\mathrm{d} h}{\mathrm{~d} t}\right| \frac{\mathrm{d} h}{\mathrm{~d} t}
$$



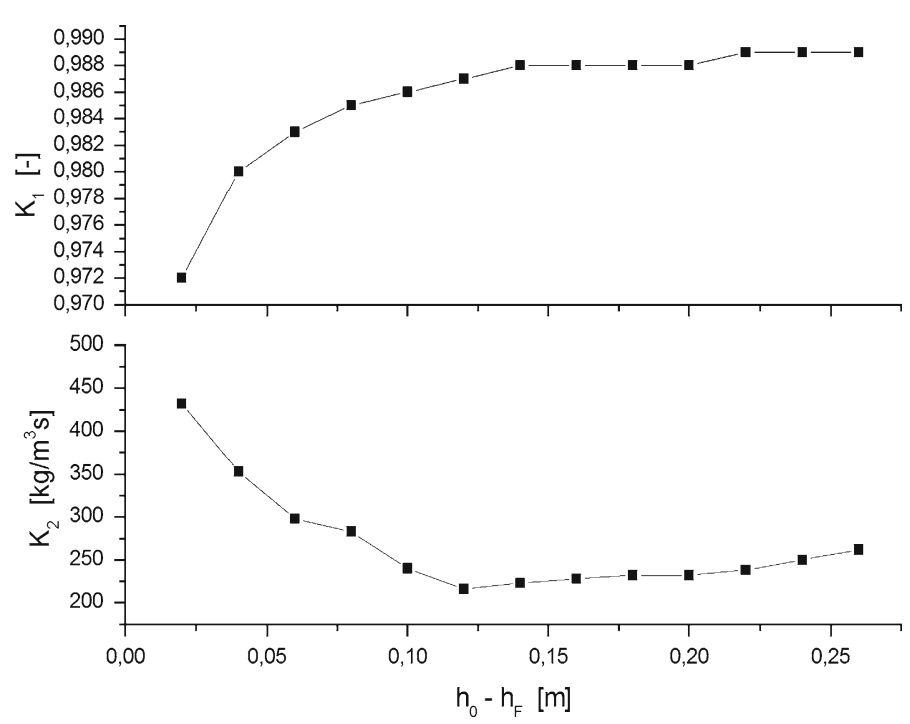

Fig. 4 Macroscopic parameters of liquid interaction with tube walls $K_{1}$ and $K_{2}$ for the applied U-tube system as the functions of initial level of liquid

where $D_{e}=\pi R+2 h_{F}$ is the length of liquid column along axis $\zeta$. The equation (9) supplemented with initial conditions (initial height and zero velocity) and solved by Runge-Kutta method allowed the pressure to be found at point 2 of the U-tube system. The experiments performed with help of the system shown in Fig. 1 (without the porous sample) provided data for pressure associated with the oscillations. A numerical optimization within Matlab environment led to the identification of parameters $K_{1}, K_{2}$ and $K_{3}$, depending on initial liquid level $h_{0}$. Since the last term is mainly associated with turbulent flow, which is rather expected for large amplitude oscillations, and since the oscillations for the U-tube system including porous material are significantly limited, the nonlinear term was neglected in the identification of drag parameters of flow in porous materials. As a result, the interaction of liquid with tube walls is represented by parameters $K_{1}$ and $K_{2}$, and their dependence on initial height of liquid is shown in Fig. 4.

While the value of $K_{1}$ is close to one and changes insignificantly with initial liquid level (maximum $3 \%$ ), the variations of $K_{2}$ are significant and must be incorporated in the model to properly express the interaction of liquid with tube walls.

\subsection{Parametric Studies}

In order to evaluate the sensitivity of pressure $p_{2}$ to the considered drag parameters of flow in porous material, the basic parametric studies are performed and their results are presented in Fig. 5. The results are obtained by numerical solution of Eqs. (7) and (8) for $h_{F}=280 \mathrm{~mm}$, $h_{0}=100 \mathrm{~mm}$, and assuming $\rho=1,000 \mathrm{~kg} / \mathrm{m}^{3} ; g=9.81 \mathrm{~m} / \mathrm{s} ; \mu=0.001$ Pas, $n=0.9$. The calibration parameters representing interaction with tube walls were selected based on data shown in Fig. 4 , i.e., $K_{1}=0.986, K_{2}=240 \mathrm{~kg} / \mathrm{m}^{3}$ s. The ranges of parameters $k$ and $\beta$ for which the analysis is presented correspond to the type of materials which are studied in the paper, i.e., foams.

It can be noticed that the parameters $k$ and $\beta$ have similar influence on shape of pressure distribution except the fact that the role of the latter parameter decreases more 

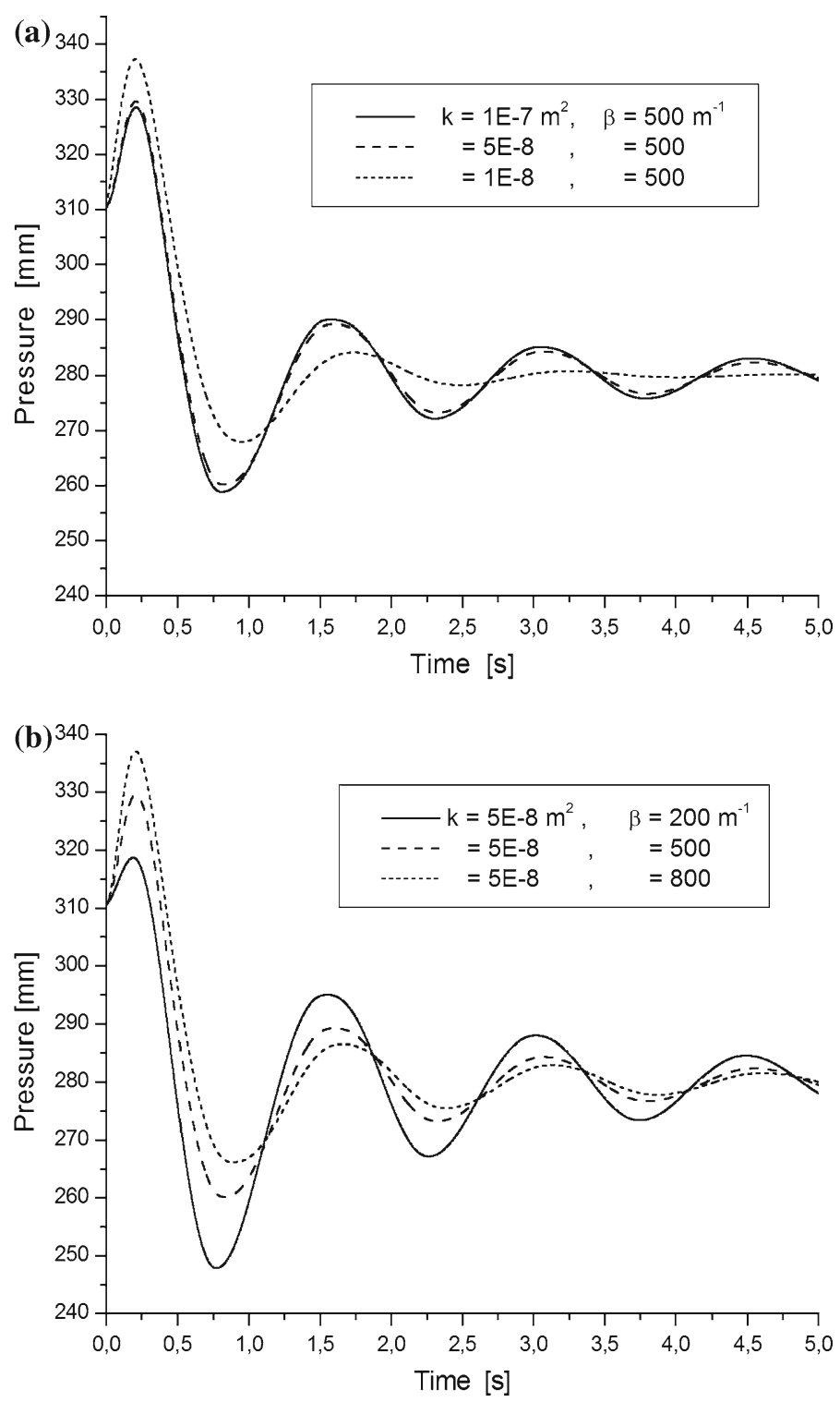

Fig. 5 Influence of permeability (a) and nonlinear drag parameter (b) on pressure oscillations in U-tube

with the decrease in amplitude of oscillations (time) than it is for the former parameter. Another observation is that the larger friction forces (corresponding to smaller $k$ and larger $\beta$ ) are considered the higher values of the amplitude in the first half period of oscillations appear. In opposite way, amplitudes of pressure for the rest of time depend on the value of the drag forces. From Fig. 5a, it also results that the linear term has limited influence on pressure when the nonlinear term assumes value $\beta=500 \mathrm{~m}^{-1}$, and then increase in $k$ from $5 \times 10^{-8} \mathrm{~m}^{2}$ to $1 \times 10^{-7} \mathrm{~m}^{2}$ does not change the values of pressure significantly. 


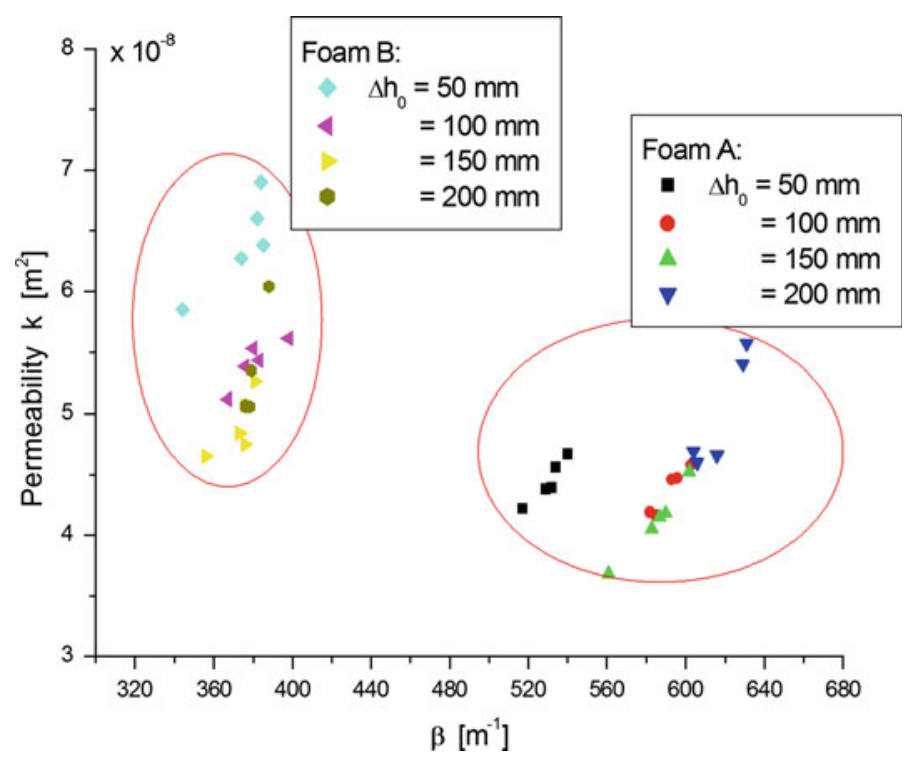

Fig. 6 Results of identification of permeability $k$ and nonlinear drug parameter $\beta$ for two open cell foams. Different initial liquid levels $\Delta h_{0}=h_{0}-h_{F}$ were applied

\subsection{Procedure and Results of Identification for Foams}

The drag parameters of flow in porous material are found by fitting the model predictions for pressure in oscillating liquid in the U-tube system to results of measurements. The objective function for the considered identification problem was chosen as:

$$
E(k, \beta)=\min \sum_{i}\left[p_{2}^{\mathrm{m}}\left(t_{i}, k, \beta\right)-p_{2}^{\mathrm{e}}\left(t_{i}\right)\right]^{2}
$$

where $p_{2}^{\mathrm{m}}$ and $p_{2}^{\mathrm{e}}$ are the values of the pressure $p_{2}$ predicted by the model and determined from experiment, respectively, for selected set of time instants $t_{i}$. Since in the initial stage of the experiments (stage of strong presence of inertial forces), the pressure measured by the transducer has nonphysical oscillations. The pressure values in this range are removed from the data taken for the minimization of the error function (10).

The identification procedure of parameters $k$ and $\beta$ is developed within Matlab environment using numerical solution of Eqs. (7) and (8) and applying global optimization with the error function (10) based on the pattern search method.

In Fig. 6, the results of identification for two open cell foams A and B, described in Sect. 4.1, are shown while the initial liquid level $h_{0}$ amounts 50, 100, 150 and $200 \mathrm{~mm}$ above the equilibrium level $h_{F}$.

Scattering of the results is visible and appears both when data for different runs from approximately the same initial liquid level as well as from different initial levels $\left(h_{0}\right)$ are exploited. Removing results for the lowest and highest $h_{0}$ (i.e., $h_{0}=50 \mathrm{~mm}$ and $h_{0}=$ $200 \mathrm{~mm}$ ), we can estimate narrow ranges of the drag parameters as following: for foam A: $k=$ $(4.0 \div 4.5) \times 10^{-8} \mathrm{~m}^{2}$ and $\beta=(560 \div 620) \mathrm{m}^{-1}$ and for foam $\mathrm{B}: k=(4.5 \div 5.5) \times 10^{-8} \mathrm{~m}^{2}$ and $\beta=(360 \div 400) \mathrm{m}^{-1}$. The results are consistent in the sense that when the material 

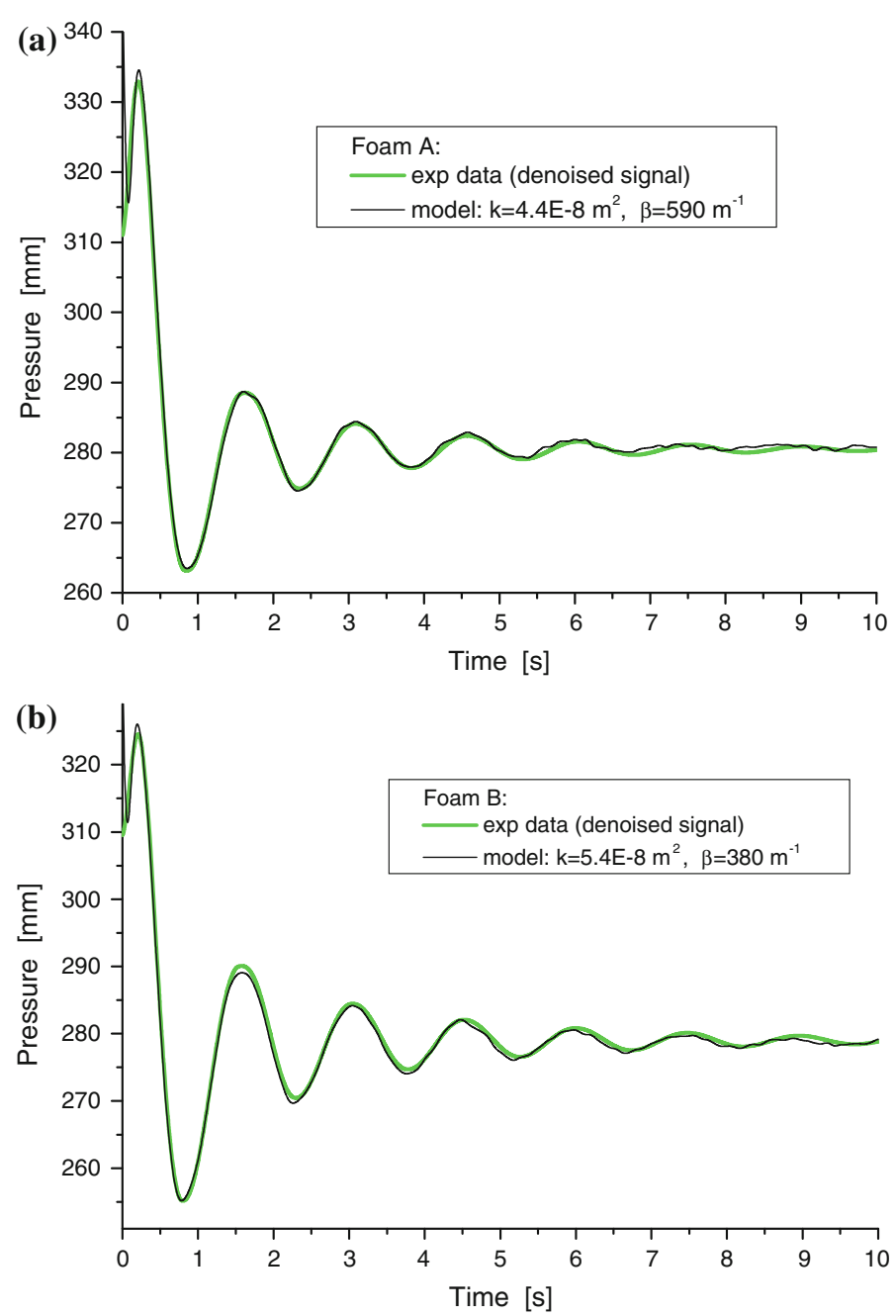

Fig. 7 Oscillations of pressure from experiments (denoised signal) and model predictions assuming the identified drag parameters

has higher $k$, it has also lower $\beta$, which is justified from the physical point of view assuming similar microstructure of foams.

In order to illustrate the quality of fitting of the experimentally determined pressure oscillations by the considered models, taking into account the identified drag parameters, in Fig. 7 , the corresponding results for foams A and B are compared. The denoised signals from experimental data are used for comparison.

\section{Conclusions}

It was shown that the developed method gives the opportunity to identify linear and nonlinear drag parameters of liquid flow in high permeability porous materials using the U-tube 
technique. The experimental data are obtained by the simple measurements of liquid pressure. The considered model incorporates the linear and nonlinear liquid interactions with porous material as well as the two linear forces representing liquid interactions with tube walls. The advantages of the U-tube technique are its simplicity and the possibility of multiple repetition of tests in the same conditions. This gives opportunity to make tests fast for optimum measurement conditions (for instance initial height). The technique can be used both for large and very small (but representative) samples because there is no problem to control very large flow velocities or small pressure gradients.

The main improvements of the proposed approach as compared to the work by Rehbinder (1992) are related to better represented physics of interaction (the latter model includes only the linear interaction between liquid and porous sample) and the application of easier way to monitor oscillations. The measurements of liquid pressure are more available source of data than measurements of liquid level, although the approach requires more complex model to fit the experimental results.

Acknowledgments This work was supported by the National Science Centre in Poland under grants 2011/01/B/ST7/04498 and 2011/01/B/ST8/07283.

Open Access This article is distributed under the terms of the Creative Commons Attribution License which permits any use, distribution, and reproduction in any medium, provided the original author(s) and the source are credited.

\section{References}

Antohe, B.V., Lage, J.L., Price, D.C., Weber, R.M.: Experimental determination of permeability and inertia coefficients of mechanically compressed aluminum porous matrices. J. Fluid Eng. Trans. ASME 119, 404-412 (1997)

Bhattacharya, A., Calmidi, V.V., Mahajan, R.L.: Thermophysical properties of high porosity metal foams. Int. J. Heat Mass Transf. 45, 1017-1031 (2002)

Hassanizadeh, M.S., Gray, W.G.: High velocity flow in porous media. Transp. Porous Media 2, 521-531 (1987)

Kaczmarek, M.: Role of inertia in falling head permeability test. Int. J. Numer. Anal. Methods Geomech. 33, 1963-1970 (2009)

Kaczmarek, M., Pakuła, M., Drelich, R.: Liquid oscillations in U-tube. Studies in Terms of Pressure (2013) (Paper submitted for publication)

Rehbinder, G.: Measurement of the relaxation time in Darcy flow. Transp. Porous Media 8, 263-275 (1992)

Sharma, S., Siginer, D.A.: Permeability measurement methods in porous media of fiber reinforced composites, Appl. Mech. Rev. 63, 020802-1-19 (2010)

Zeng, Z., Grigg, R.: A criterion for non-Darcy flow in porous media. Transp. Porous Media 63, 57-69 (2006) 